Alegría Cabrera ${ }^{1}$

Carmen Trigo ${ }^{1}$

Lucía Cox ${ }^{1}$

Rafael Celis ${ }^{1}$

Juan Cornejo ${ }^{1}$

${ }^{1}$ Instituto de Recursos Naturales y Agrobiología de Sevilla (IRNAS), CSIC, Sevilla, Spain.

\section{A Comparative Study of the Use of Organoclay- Based Formulations and Organic Amendment to Reduce the Leaching of the Herbicide MCPA in Soil}

\begin{abstract}
MCPA (4-chloro-2-methylphenoxyacetic acid) is an acidic herbicide widely used on olive crops in Spain. Due to its anionic form at natural soil $\mathrm{pH}$, there is high risk of leaching and groundwater contamination by the use of this herbicide. The aim of this work was to study the effects of organoclay-based formulations of MCPA and olive oil waste amendment on MCPA leaching in a sandy loam soil. For this purpose, batch adsorption and column leaching studies were performed. The organoclays used to prepare the clay-based formulations of MCPA were obtained by treating Wyoming montmorillonite (SWy-2) and Arizona montmorillonite (SAz-1) with an amount of hexadecyltrimethylammonium (HDTMA) cation equal to $100 \%$ of the CEC of the montmorillonites. The organic residue used in this study was a solid waste from olive oil production (olive oil waste, OOW). The soil was amended with the organic residue at the rate of $10 \%(\mathrm{w} / \mathrm{w})$. Batch release and column leaching studies indicated that organoclay-based formulations of MCPA reduced the release rate and the leaching of the herbicide as compared to the use of a conventional formulation containing the herbicide in an immediately available form. The increase in soil organic matter of the soil upon amendment with the organic residue also resulted in greater adsorption and reduced leaching of MCPA in the soil. Accordingly, both the use of organoclaybased formulations and the amendment of soil with OOW are proposed as efficient strategies to reduce extensive leaching losses associated with the application of MCPA in high-risk scenarios, such as Mediterranean olive groves.
\end{abstract}

Keywords: Herbicide leaching; MCPA; Olive oil waste; Organoclays; Soil

Received: July 17, 2008; revised: September 10, 2008; accepted: October 1, 2008

DOI: 10.1002/clen.200800159

\section{Introduction}

Surface and groundwater contamination by herbicides used in olive groves in Spain and other Mediterranean countries has become a serious issue, and has led authorities to prohibit or restrict the application of widely used active ingredients, e.g., simazine, diuron, terbuthylazine, or MCPA [1]. In olive groves, conditions that favor offsite movement of the applied herbicides predominate. Soil texture, pronounced slopes, and climatic conditions favor losses of herbicide by leaching and runoff processes. To compensate for such losses, herbicides need to be applied in concentrations greatly exceeding those strictly required for weed control, and the excessive quantities added increase the risk of surface and groundwater pol-

Correspondence: Prof. J. Cornejo, Instituto de Recursos Naturales y Agrobiología de Sevilla (IRNAS), CSIC, Avenida Reina Mercedes 10, Apartado 1052, 41080 Sevilla, Spain.

E-mail: cornejo@irnase.csic.es

Abbreviations: HDTMA, Hexadecyltrimethylammonium; MCPA, 4chloro-2-methylphenoxyacetic acid; OOW, Olive oil waste; SA-HDTMA, HDTMA-treated Arizona montmorillonite; SW-HDTMA, HDTMA-treated Wyoming montmorillonite lution. In this context, there is a need to develop strategies directed to preventing water pollution by herbicides applied to olive groves, particularly in high-risk scenarios such as Mediterranean areas [2].

The use of slow release formulations has been demonstrated to be an efficient strategy to reduce the environmental impact of soilapplied pesticides. Beneficial effects related to the use of slow release formulations include reduced mobility of the active ingredient, reduction in the amount of chemical required for pest control, savings in manpower and energy from the reduction of the number of applications required in comparison to conventional formulations, increased safety for the pesticide applicator, and a general decrease in non-target effects [3]. Organoclays, i.e., natural clay minerals with their original inorganic exchange cations replaced with organic cations, have been shown to be very good adsorbents for different classes of pesticides, and therefore, have been proposed as carriers for the design of slow release formulations of pesticides [4-7].

Soils of low organic carbon content have a low capacity for retarding pesticide mobility [8], since soil organic matter is the primary adsorbent for pesticides and adsorption is one of the main processes reducing the level of pesticide leaching in soil [9]. Organic amendments can be used to modify the surfaces of soils and subsurface 
materials, promoting adsorption and reducing groundwater contamination by pesticides $[10,11]$. Furthermore, the addition of organic amendments modifies soil chemical and biological conditions, which can affect degradation and, in some cases, accelerate this process [11-13], reducing the total amount of chemical potentially available for leaching. The new technologies for olive-oil extraction generate a solid residue rich in organic matter. This olive oil waste (OOW) is generated in great amounts in Spain $(4,000,000$ $\mathrm{Mg} /$ year), and represents a major environmental problem. Soil incorporation of OOW has been shown to increase fertility and control erosion [14, 15], but also to reduce pesticide leaching [16].

In this work, batch and column leaching experiments were designed to assess and compare the ability of organoclay-based formulations and the application of OOW to soil to reduce the leaching of the herbicide MCPA in a Mediterranean sandy loam soil. MCPA was selected since it is a highly mobile herbicide commonly used in high risk scenarios of surface and groundwater contamination, such as Mediterranean olive groves.

\section{Materials and Methods}

\subsection{Herbicide}

Analytical grade MCPA (purity = 98.9\%) from Riedel-de Haën (Germany) was used to prepare the clay-based formulations of the herbicide, the initial herbicide solutions used in the adsorption experiments, and the external standards for herbicide analysis. Commercial MCPA, amine salt 60\% soluble concentrate (SL) from Tratamientos Guadalquivir S.L. (Spain), was used as a reference standard formulation in the release and leaching experiments.

\subsection{Soil and Organic Amendment}

The soil used in the experiments was a sandy loam soil $(0-20 \mathrm{~cm})$ with $73 \%$ sand, $7 \%$ silt and $20 \%$ clay sampled from an experimental olive field located in Seville (Spain). It contained 1.0\% $\mathrm{CaCO}_{3}$ and $1.62 \%$ organic matter. The $\mathrm{pH}$ measured in a $1: 2(\mathrm{w} / \mathrm{w})$ soil:deionized water suspension was 8.2. The organic amendment used was a fresh, uncomposted olive oil waste (OOW). The properties of this organic waste are: $\mathrm{pH} 5.8,93.2 \%$ organic matter, $25 \mathrm{~g} / \mathrm{kg}$ dissolved organic carbon, and a $\mathrm{C} / \mathrm{N}$ ratio of 18.3 . The soil was amended in the laboratory with OOW at the rate of $10 \%(\mathrm{w} / \mathrm{w})$.

\subsection{Organoclays and Organoclay-Based Formulations of MCPA}

The organoclays used in this work, i.e., SW-HDTMA and SA-HDTMA, were prepared by treating Na-rich SWy-2 Wyoming montmorillonite and Ca-rich SAz-1 Arizona montmorillonite with an ethanol/
$\mathrm{Cl}=\mathrm{CH}_{3}-\mathrm{CH}_{2} \mathrm{COOH}$

MCPA

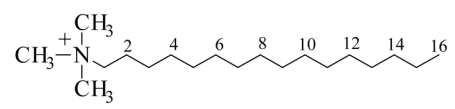

HDTMA
Figure 1. Chemical structures of MCPA and HDTMA.

water (50:50) solution containing an amount of hexadecyltrimethylammonium (HDTMA) cation, Fig. 1, as the chloride salt, equal to the cation exchange capacity (CEC) of the montmorillonites [6]. The CEC of SWy-2 and SAz-1 are 74.6 and $120 \mathrm{cmol}_{\mathrm{c}} / \mathrm{kg}$, respectively. Additional physicochemical properties of SWy-2 and SAz-1 can be found in van Olphen and Fripiat [17]. The suspensions were shaken for $24 \mathrm{~h}$, centrifuged, washed with distilled water until $\mathrm{Cl}$-free, and then lyophilized. Some characteristics of the resultant organoclays are given in Tab. 1. The percentage of the CEC occupied by HDTMA cations (OCtS) was calculated from the N-content of the samples, determined by elemental analysis. The basal spacing values, $d_{001}$, were measured on air-dried oriented specimens in a Siemens D5000 diffractometer using CuKa radiation.

The organoclay-based formulations of MCPA were prepared by treating $400 \mathrm{mg}$ of organoclay (SW-HDTMA or SA-HDTMA) with 160 $\mathrm{mL}$ of an aqueous solution of MCPA. The concentration of MCPA in solution was $600 \mathrm{mg} / \mathrm{L}$ for SW-HDTMA and $200 \mathrm{mg} / \mathrm{L}$ for SA-HDTMA, in order to achieve similar herbicide content in both formulations. Extractions with a 60:40 methanol:diluted $\mathrm{H}_{3} \mathrm{PO}_{4}(\mathrm{pH}=2)$ mixture revealed that the final amounts of MCPA adsorbed on SW-HDTMA and SA-HDTMA were 6.5 and 5.6\%, respectively.

\subsection{Batch Adsorption Isotherms}

Adsorption isotherms of MCPA on the organoclays and on unamended and amended soil were obtained by the batch equilibration procedure. MCPA adsorption isotherms on the organoclays were obtained by shaking suspensions containing $20 \mathrm{mg}$ of organoclay and $8 \mathrm{~mL}$ of MCPA aqueous solutions with initial herbicide concentrations ranging between 0.1 and $2 \mathrm{mg} / \mathrm{L}$ at $20 \pm 2^{\circ} \mathrm{C}$ for $24 \mathrm{~h}$. MCPA adsorption isotherms on unamended and amended soil were obtained in a similar manner by equilibrating $5 \mathrm{~g}$ of soil with $10 \mathrm{~mL}$ of MCPA solutions with initial herbicide concentrations ranging between 1 and $10 \mathrm{mg} / \mathrm{L}$. After equilibration, the suspensions were centrifuged and $4 \mathrm{~mL}$ of the supernatant solutions was removed for analysis. The herbicide concentration in the supernatant solutions, $C_{\mathrm{e}}$, was determined by high-performance liquid chromatography (HPLC) as described below. The amount of herbicide adsorbed in the soil and the organoclays, $C_{\mathrm{s}}$, was calculated from the difference between the initial and the equilibrium solution concentrations.

Table 1. Some characteristics of the organoclays used as adsorbents of MCPA.

\begin{tabular}{lllr}
\hline Organoclay & Montmorillonite & Organic cation & OCtS \\
\hline SW-HDTMA & SWy-2 & Hexadecyltrimethylammonium & 93 \\
SA-HDTMA & SAz-1 & Hexadecyltrimethylammonium & 100 \\
\hline
\end{tabular}

a) Organic cation saturation: percentage of the CEC of the montmorillonites compensated by HDTMA cations (calculated from the N content of the organoclays)

b) $d_{001}$ : basal spacing value for air-dried oriented specimens. 


\subsection{Kinetics of MCPA Release from the Organoclay- Based Formulations}

Ten milligrams of organoclay-based formulations of MCPA were added to $250 \mathrm{~mL}$ of distilled water in glass bottles sealed with screw caps. At selected times, the bottles were hand-shaken, allowed to settle for $10 \mathrm{~min}$, and then $3 \mathrm{~mL}$ of the supernatant solution was removed, filtered, and analyzed by HPLC to determine the MCPA concentration. The aqueous concentrations of MCPA expected assuming complete release of MCPA from SW-HDTMA and SAHDTMA were 2.6 and $2.2 \mathrm{mg} / \mathrm{L}$, respectively. These concentrations would be at the high end in comparison to those used in the batch adsorption isotherms. The release kinetics of MCPA from the standard commercial formulation was also measured for comparison purposes.

\subsection{Column Leaching Experiment}

Leaching was studied in glass columns of length $30 \mathrm{~cm}$ and i.d. 3.1 $\mathrm{cm}$. The top $5 \mathrm{~cm}$ was filled with sea sand and the bottom $5 \mathrm{~cm}$ with sea sand plus glass wool, to prevent losses of soil during the experiment. The other $20 \mathrm{~cm}$ was hand packed with air-dried soil, either unamended or amended with OOW at a rate of $10 \%$, which was then saturated with deionized water from the top, and allowed to drain for $24 \mathrm{~h}$. The calculated pore volume of the columns after saturation was ca. $60 \mathrm{~mL}$. The amount of MCPA corresponding to an application rate of $2 \mathrm{~kg} / \mathrm{ha}(0.15 \mathrm{mg}$ a.i.) was applied to the top of duplicate unamended soil columns either as organoclay-based formulations or as the commercial standard formulation. In a separate set of experiments, the commercial standard formulation of MCPA was added to the top of duplicate columns filled with either unamended or OOW-amended soil. Daily, $15 \mathrm{~mL}$ of deionized water was added to the top of the columns, the leachates were collected, and the concentration of MCPA in the leachates was determined by HPLC.

\subsection{Herbicide Analysis}

The herbicide MCPA was analyzed by HPLC using a Waters 600E chromatograph coupled to a Waters 996 diode-array detector. The analytical conditions were: Novapack C18 column $(150 \mathrm{~mm}$ length $\cdot 3.9 \mathrm{~mm}$ i.d.), eluent mixture, 60:40 methanol:diluted $\mathrm{H}_{3} \mathrm{PO}_{4}$ $(\mathrm{pH}=2)$ at a flow rate of $1 \mathrm{~mL} / \mathrm{min}, 25 \mu \mathrm{L}$ injection volume, and $\mathrm{UV}$ detection at $230 \mathrm{~nm}$. External calibration curves with standard solutions between 0.1 and $10 \mathrm{mg} / \mathrm{L}$ were used in the calculations.

\section{Results and Discussion}

\subsection{Characteristics of the Organoclays}

The amounts of hexadecyltrimethylammonium (HDTMA) present in the organoclays, Tab. 1, were calculated from the N-content of the samples, and were found to be close to the amounts of organic cation added during the synthesis. This reflects the success of the exchange reaction, as expected from the high affinity of montmorillonites for large alkylammonium cations such as HDTMA [18]. The basal spacing values, $d_{001}$, of air-dried oriented specimens of the organoclays, Tab. 1, indicated that the arrangement of HDTMA cations in the interlayers of SWy-2 and SAz-1 is dependent on the surface charge density of the montmorillonite. Thus, HDTMA formed

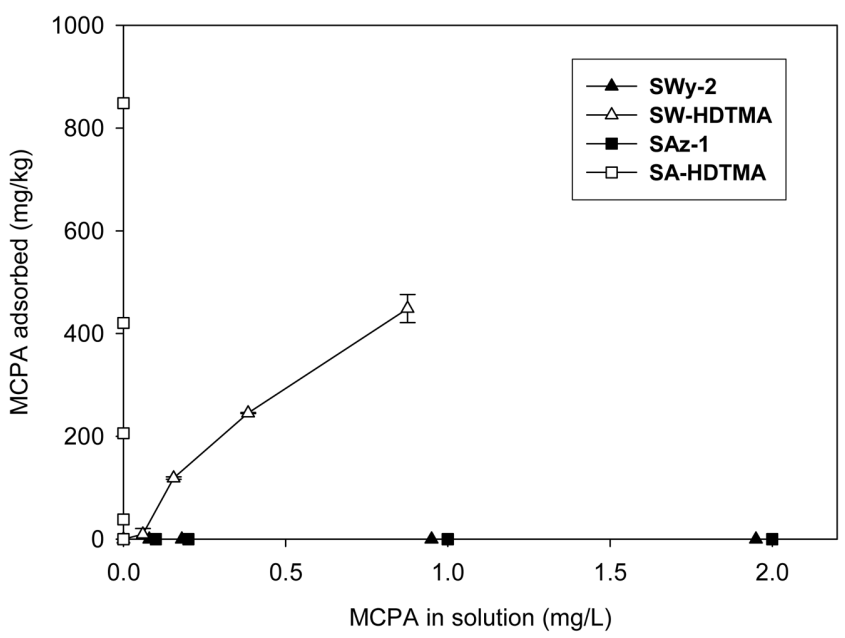

Figure 2. Adsorption isotherms of MCPA on organoclays (SW-HDTMA and SA-HDTMA) and unmodified clays (SWy-2 and SAz-1). Data were extracted from Celis et al. [2].

horizontal bilayers in the low-charge montmorillonite SWy-2 $\left(d_{001} \approx\right.$ $18 \AA$ A), whereas it formed a paraffinic structure, with vertical arrangement, in the high-charge montmorillonite SAz-1 $\left(d_{001}>22 \AA\right)$ $[19,20]$. Previous work has shown that large organic cations, e.g., HDTMA are strongly retained in the interlayers of montmorillonites. Solvents do not significantly displace the organic cation from the clay, and the structure is stable in the presence of high concentrations of metal cations [18].

\subsection{Adsorption of MCPA on the Organoclays and Release from the Organoclay-Based Formulations}

The organoclays used in this work (SW-HDTMA and SA-HDTMA) were selected on the basis of a preliminary adsorption-desorption study revealing their high affinity for the herbicide MCPA, see Fig. 2 , and the low hysteresis of the adsorption-desorption isotherm found for SW-HDTMA, which are desirable features for the use of clays as supports for the slow release of pesticides [2]. In contrast, adsorption of MCPA by the untreated clays (SWy-2 and SAz-1) was negligible due to repulsions between the herbicide and the negatively charged clay surfaces, see Fig. 2. Replacement of the original inorganic exchange cations of SWy-2 and SAz- 1 by HDTMA cations changes the nature of the montmorillonite surfaces from hydrophilic to hydrophobic, increasing their affinity for the herbicide MCPA. This behavior has also been observed for other acidic pesticides, such as 2,4-D, dicamba or imazamox [21-23]. The higher affinity of SA-HDTMA for MCPA compared to that displayed by SWHDTMA can be explained by the paraffinic $\left(d_{001}>22 \AA\right)$ structure resulting from incorporation of HDTMA cations in SAz-1, which creates a wide interlayer organic phase with a strong affinity for herbicides [2].

The release kinetics of MCPA into water from formulations prepared by preadsorbing the herbicide on SW-HDTMA and SA-HDTMA, are shown in Fig. 3. In contrast to the reference standard formulation, which released $100 \%$ of the herbicide instantaneously, the organoclay-based formulations of MCPA displayed slow release properties in water. The initial amount of MCPA released by SW- 


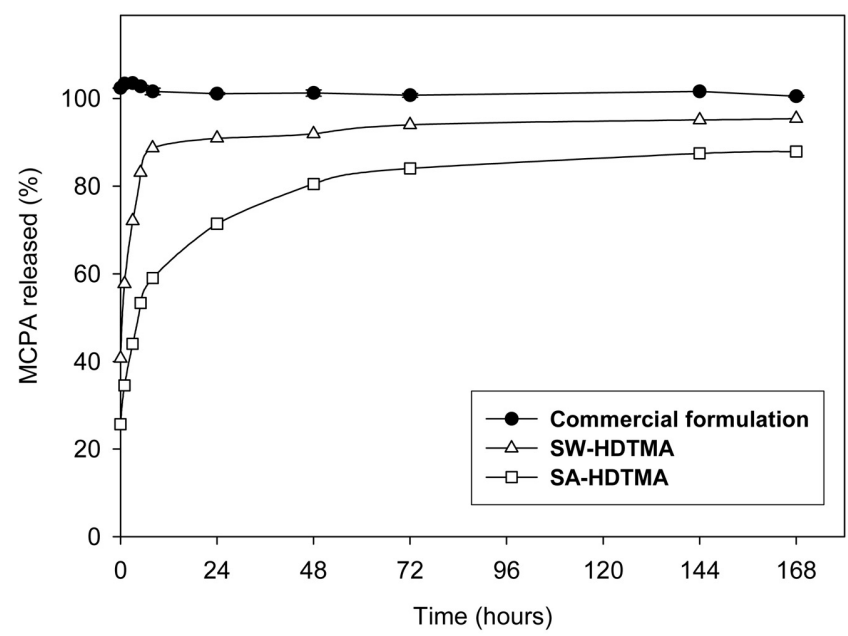

Figure 3. Release kinetics of MCPA into water from organoclay-based formulations and from a reference standard commercial formulation.

HDTMA was $41 \%$, whereas the final amount of MCPA released by this organoclay was 95\% after $168 \mathrm{~h}$. For SA-HDTMA, the initial release was $26 \%$, whereas the final release was $88 \%$ after $168 \mathrm{~h}$. Both the release rate and the total amount of herbicide released at the end of the experiment was less for SA-HDTMA compared to SWHDTMA, which is consistent with the different affinities of the organoclays for the herbicide, see Fig. 2. Since different rates and extents of MCPA occurred depending on the nature of the adsorbent, the possibility exists to select the most suitable organoclay to achieve the desired release behavior of MCPA.

\subsection{Effect of the Organic Amendment on the Adsorption of MCPA by the Soil}

The adsorption isotherms of MCPA on unamended soil and soil amended with OOW are shown in Fig. 4. Adsorption isotherms were fitted to the Freundlich equation and adsorption coefficients were calculated, see Fig. 4. MCPA adsorption by the unamended soil was low, due to the anionic character of the herbicide. The organic amendment (OOW) increased considerably the adsorption capacity of the soil for the herbicide MCPA, see Fig. 4, which can be attributed to the increase in organic matter content of the soil upon amendment (from 1.6 to $4.3 \%$ ). Similar results were observed in a previous study with the anionic herbicide clopyralid and a soil amended with olive-mill wastewater [11].

\subsection{Column Leaching Experiments}

The effects of formulation and OOW addition on the breakthrough curves (BTCs) of MCPA in soil columns are shown in Fig. 5. Both the application of the herbicide as organoclay complexes on unamended soil and the amendment of the soil with the organic residue clearly resulted in lower MCPA concentration in leachates, flattening of the BTCs, and a lower total amount of herbicide leached compared to the application of the commercial formulation to unamended soil columns. The application of organoclaybased formulations of MCPA also resulted in the shifting of the maximum concentration peak of the BTC to larger water volumes compared to the commercial formulation.

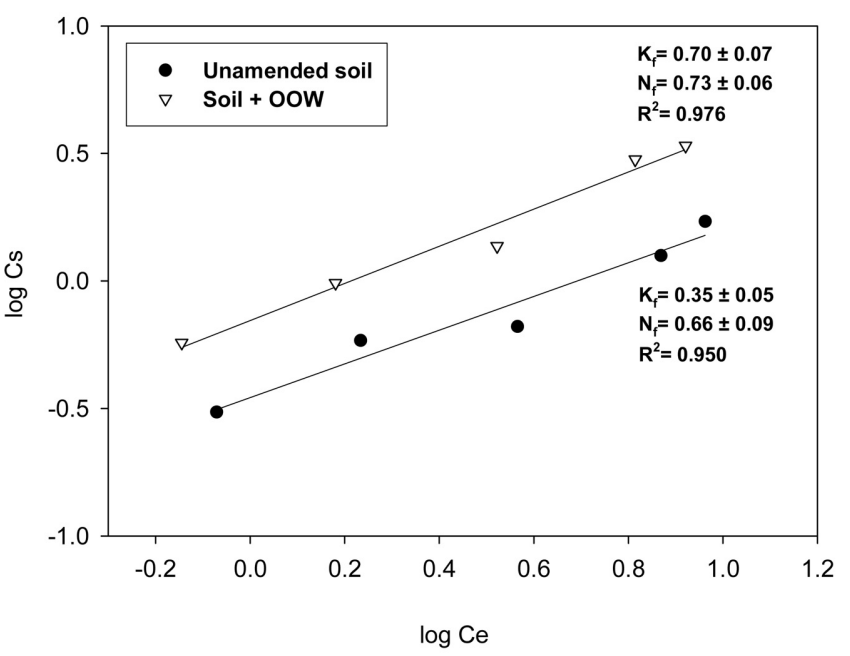

Figure 4. Freundlich adsorption isotherms of MCPA on unamended soil and soil amended with olive oil waste (OOW). Freundlich adsorption coefficients for each isotherm are included in the graph.

Regarding the effect of formulation, the maximum concentration of MCPA in leachates decreased in the order: commercial > SWHDTMA > SA-HDTMA, which is in accordance with the affinity of the organoclays for the herbicide and the herbicide release rates observed in the batch release experiments, see Figs. 2 and 3. A very interesting feature from Fig. 5 is that for the organoclay-based formulations of MCPA, especially for SA-HDTMA, the presence of the herbicide in the leachates remained moderate after larger volumes of water added, as compared to the commercial formulation, demonstrating the slow release behavior of the formulations.

The organic amendment, OOW, also reduced the level of MCPA leaching and led to moderate concentrations of MCPA in leachates, as can be seen from the cumulative and relative BTCs, see Fig. 5, even though the maximum of the BTC occurred at the same time in unamended and amended soil and the appearance of MCPA in leachates occurred slightly earlier in amended soil. The latter observation can be attributed to the very high content in dissolved organic carbon (DOC, $25 \mathrm{~g} / \mathrm{kg}$ ) of the OOW, which could have initially favored leaching by formation of soluble DOC-MCPA complexes. Nevertheless, the total amount of MCPA leached from amended soil columns was much less than that leached from unamended soil, as a result of the increase in the adsorption capacity of the soil upon amendment with the organic residue.

\section{Concluding Remarks}

The results of this work show that both the use of organoclay-based formulations and the amendment of soil with olive oil waste (OOW) can be efficient strategies to reduce extensive leaching losses associated with the application of highly mobile herbicides, such as MCPA, in high-risk scenarios, such as Mediterranean olive groves. The reduced release rate of MCPA from organoclay-based formulations, compared to its immediate release from conventional commercial formulations, results in lower herbicide concentration in leachates and lower total amount of herbicide leached from soil columns. The enhanced adsorption of MCPA provided by the addition of OOW to soil also resulted in reduced herbicide leaching, and OOW addition to soil would have the added value of being an alter- 

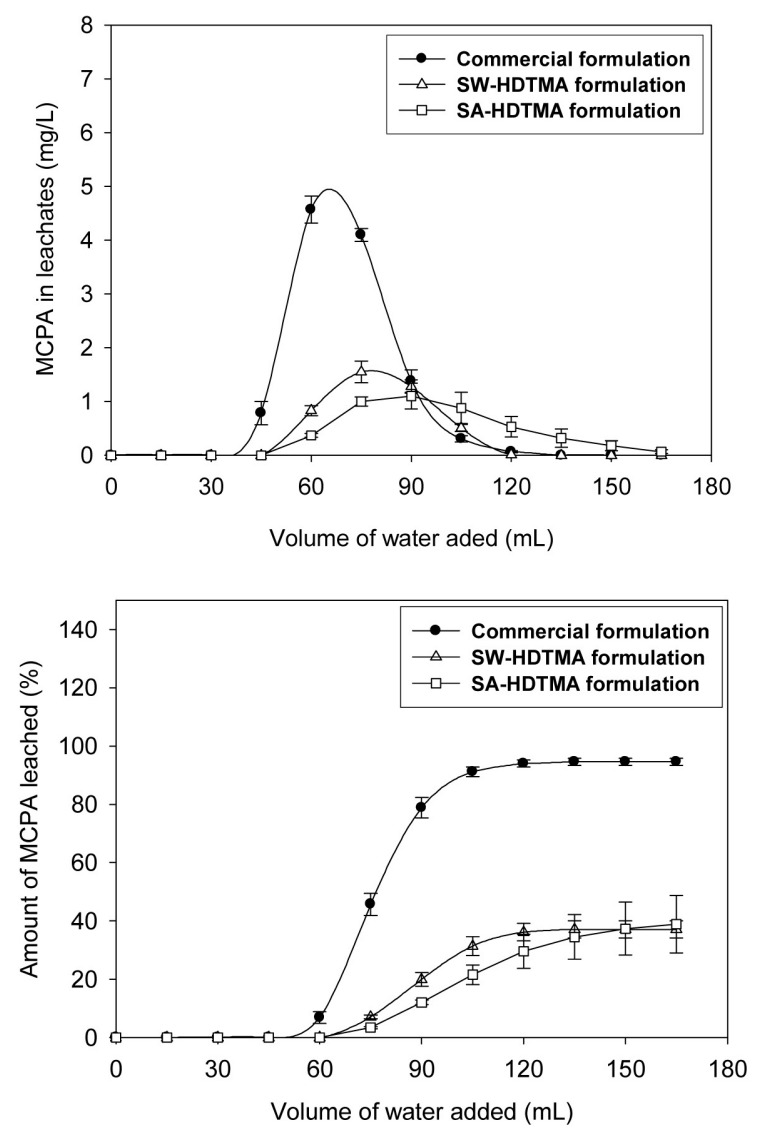
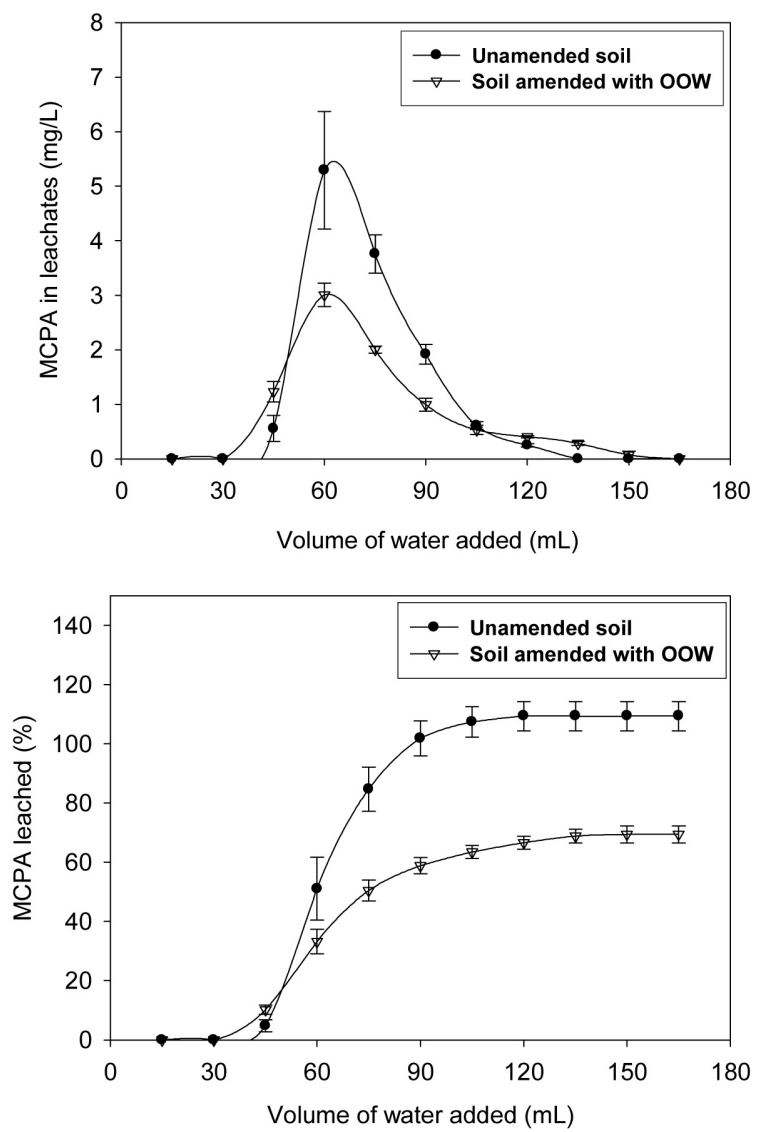

Figure 5. Relative (top) and cumulative (bottom) breakthrough curves of MCPA in soil columns as affected by formulation and organic amendment addition. The effect of formulation was studied on unamended soil, whereas the effect of OOW addition was studied using the commercial formulation of MCPA.

native for OOW disposal. However, when using this practice, the possibility of an enhanced initial leaching, due to the presence of dissolved organic carbon in the organic residue, should also be considered.

\section{Acknowledgments}

This work has been financed by the Spanish Ministry of Education and Science (MEC/FEDER) through the projects AGL2007-65771-C0201 and AGL2008-04031-C02-01, and by Junta de Andalucía through the projects P06-AGR-1565 and P07-AGR-03077. The authors wish to thank Tratamientos Guadalquivir S.L. for kindly supplying the commercial formulation of MCPA. Carmen Trigo and Alegría Cabrera also thank the Spanish Ministry of Education and Science for their FPI fellowships.

\section{References}

[1] R. Santiago-Mora, F. Martin-Laurent, R. de Prado, A. R. Franco, Degradation of simazine by microorganisms isolated from soils of Spanish olive fields, Pest Manage. Sci. 2005, 61, 917.

[2] R. Celis et al., Selective modification of clay minerals for the adsorption of herbicides widely used in olive groves, J. Agric. Food Chem. 2007, 55, 6650 .
[3] Z. Gerstl, A. Nasser, U. Mingelgrin, Controlled release of pesticides into soils from clay-polymer formulations, J. Agric. Food Chem. 1998, 46, 3797.

[4] Y. El-Nahhal, S. Nir, L. Margulies, B. Rubin, Reduction of photodegradation and volatilization of herbicides in organo-clay formulations, Appl. Clay Sci. 1999, 14, 105.

[5] M. C. Hermosín, M. J. Calderón, J. P. Aguer, J. Cornejo, Organoclays for controlled release of the herbicide fenuron, Pest Manage. Sci. 2001, $57,803$.

[6] R. Celis, M. C. Hermosín, M. J. Carrizosa, J. Cornejo, Inorganic and organic clays as carriers for controlled release of the herbicide hexazinone, J. Agric. Food Chem. 2002, 50, 2324.

[7] L. Cornejo et al., Use of modified montmorillonites to reduce herbicide leaching in sports turf surfaces: Laboratory and field experiments, Appl. Clay Sci., in press. DOI: 10.1016/j.clay.2008.01.008.

[8] L. Guo, T. J. Bicki, T. D. Hinesly, A. S. Felsot, Effect of carbon-rich waste materials on movement and sorption of atrazine in a sandy, coarse-textured soil, Environ. Toxicol. Chem. 1991, 10, 1273.

[9] C. T. Chiou, in Reactions and Movement of Organic Chemicals in Soils, SSSA Special Publication No. 22, Madison, WI 1989, p. 1.

[10] E. Barriuso, S. Houot, C. Serra-Wittling, Influence of compost addition to soil on the behavior of herbicides, Pestic. Sci. 1996, 49, 65.

[11] L. Cox et al., Porosity and herbicide leaching in soils amended with olive-mill waste water, Agric. Ecosys. Environ. 1997, 65, 151.

[12] E. Topp, L. Tessier, E. G. Gregorich, Dairy manure incorporation stimulates rapid atrazine mineralization in an agricultural soil, Can. J. Soil Sci. 1996, 76, 403. 
[13] J. Gan, S. R. Yates, S. Papiernik, D. Crowley, Application of organic amendments to reduce volatile pesticide emissions from soil, Environ. Sci. Technol. 1998, 32, 3094.

[14] G. Brunetti, C. Plaza, N. Senesi, Olive pomace amendment in Mediterranean conditions: Effect on soil and humic acid properties and wheat (Tritiucm turgidum L.) yield, J. Agric. Food Chem. 2005, 53, 6730.

[15] A. López-Piñeiro, J. Fernández, J. M. Rato Nunes, A. García, Response of soil and wheat crop to the application of two-phase olive mill waste to Mediterranean agricultural soils, Soil Sci. 2006, 171, 728.

[16] A. Cabrera et al., Fate of diuron and terbuthylazine in soils amended with two-phase olive oil mill waste, J. Agric. Food Chem. 2007, 55, 4828.

[17] H. van Olphen, J. J. Fripiat, Data Handbook for Clay Minerals and Other Non-Metallic Minerals, Pergamon Press, Oxford 1979.

[18] A. Moronta, in Clay Surfaces: Fundamentals and Applications (Eds: F. Wypych, K. G. Satyanarayana), Elsevier, Amsterdam 2004, p. 321.
[19] G. Lagaly, Layer charge heterogeneity in vermiculites, Clays Clay Miner. 1982, 30, 215.

[20] W. F. Jaynes, S. A. Boyd, Clay mineral type and organic compound sorption by hexadecyltrimethylammonium exchanged clays, Soil Sci. Soc. Am.J. 1991, 55, 43.

[21] M. C. Hermosín, J. Cornejo, Binding mechanism of 2,4-dichlorophenoxyacetic acid by organoclays, J. Environ. Qual. 1993, 22, 325.

[22] H. Zhao, W. F. Jaynes, G. F. Vance, Sorption of the ionizable organic compound, dicamba (3,6-dichloro-2-methoxy benzoic acid), by organoclays, Chemosphere 1996, 33, 2089.

[23] R. Celis et al., Sorption of the ionizable pesticide Imazamox by organoclays and organohydrotalcites, J. Environ. Sci. Health, Part B 1999, 34, 929. 